\title{
Evaluation of anterior pituitary hormonal profile in patients with first seizure of tonic-clonic and temporal lobe epilepsies in Al-Zahra hospital of Isfahan, Iran, during 2014-2016: comparison of hormonal profiles in 2 types of epilepsy
}

\author{
Maryam Ghasemi Aliabadi ${ }^{\circledR}$, Mohammad Reza Najafi², Rokhsareh Meamar ${ }^{3,4^{*}}{ }^{\circledR}$, Shahrzad Nematollahi ${ }^{5}$, Noushin \\ Mehrbod $^{6}$, Mahsa Dastjerdi', Forouzan Moinzadeh ${ }^{7}$ \\ ${ }^{1}$ Medical Student, Department of Medical Science, School of Medicine, Najafabad Branch, Islamic Azad University, Najafabad, \\ Iran \\ ${ }^{2}$ Professor of Neurology, Isfahan University of Medical Sciences, Isfahan, Iran \\ ${ }^{3}$ Isfahan Neurosciences Research center, Isfahan University of Medical Sciences, Isfahan, Iran \\ ${ }^{4}$ Isfahan Clinical Toxicology Research Center, Isfahan University of Medical Sciences, Isfahan, Iran \\ ${ }^{5}$ Ph.D. student, Department of Epidemiology and Biostatistics, School of Public Health, Tehran University of Medical Sciences, \\ Tehran, Iran \\ ${ }^{6}$ Department of Neurology, Isfahan University of Medical Sciences, Isfahan, Iran \\ ${ }^{7}$ Department of pathology, Islamic Azad University, Najafabad branch, Najafabad, Isfahan, Iran
}

*Corresponding Author: Rokhsareh Meamar, M.D, Ph.D, Isfahan Neurosciences Research Center and Isfahan Clinical Toxicology Research Center, Isfahan University of Medical Sciences, Isfahan, Iran

Email: Meamar@pharm.mui.ac.ir

\begin{abstract}
Background and aims: Temporal lobe and tonic-clonic seizures are the most common types of epilepsy. It is a disease that disrupts the regulation of the hypothalamic pituitary adrenal (HPA) axis. The disturbance in the regulation of this axis affects the secretion of hormones from the anterior pituitary. Due to the different origins of these 2 types of epilepsy listed above, this study aimed to examine their differences in the level of anterior pituitary hormones.

Methods: This cross-sectional study was conducted in Al-Zahra hospital of Isfahan, Iran, from 2014 to 2016 . A total of 36 patients with the idiopathic first seizure were selected for the examination including 20 patients with tonic-clonic seizure and 16 patients with temporal lobe seizure. The diagnosis of epilepsy was confirmed by a neurologist along with electroencephalogram (EEG) findings. The levels of anterior pituitary hormones including thyroid-stimulating hormone (TSH), adrenocorticotrophic hormone (ACTH), GH (growth hormone), follicle-stimulating hormone (FSH), luteinizing hormone (LH) and PRL (prolactin) were measured and compared using ELISA method in both groups.

Results: The results indicated that all the anterior pituitary hormones except TSH and ACTH had higher means in the temporal lobe epilepsy than in the tonic-clonic epilepsy, but a significant difference (mean $\pm \mathrm{SD}$ ) was reported only in $\mathrm{GH}(4.59 \pm 2.95)(2.55 \pm 1.87)(P<0.005)$ and FSH (19.4 \pm 14.06$)(4.34 \pm 2.37)(P<0.033)$ respectively in the temporal lobe and tonic-clonic seizures. Meanwhile, there was a significant relationship between seizure, regardless of its type, and the level of growth hormone and FSH.

Conclusion: In the future, hormonal changes may be used as an adjunct to diagnose the type of epilepsy. More studies are required to confirm the findings.

Keywords: Seizure, Hormonal profile, Tonic-clonic, TLE
\end{abstract}

Received: 1 August 2017, Accepted: 24 November 2018, ePublished: 4 April 2019

\section{Introduction}

The anterior pituitary is often known as the master gland because this gland along with the hypothalamus organizes and coordinates the regulatory operations of the other endocrine glands. The anterior pituitary gland produces six major hormones called prolactin (PRL), growth hormone $(\mathrm{GH})$, adrenocorticotrophic hormone $(\mathrm{ACTH})$, luteinizing hormone (LH), follicle-stimulating hormone (FSH), and thyroid-stimulating hormone (TSH). These hormones are secreted by a pulsing pattern, indicating that they are stimulated by a series of hypothalamic releasing factors. Hypothalamus, hippocampus, parahippocampus, and amygdala are parts of the limbic system located in the temporal lobe. The amygdala is an area with a bilateral relationship with many regions of the brain, including thalamus, hypothalamus, hippocampus, parahippocampus and brain stem, and is an essential area for coordinating behaviors and intrinsic autonomic responses to environmental stimuli. The hippocampus receives sensory inputs from the neocortex, especially the limbic cortex, 
amygdala, and hypothalamus. Temporal lobe seizures typically originate from this area. Hippocampus inhibits the activity of the hypothalamic pituitary adrenal (HPA) axis. The hypothalamus receives information from the hippocampus and amygdala, and the paraventricular nucleus (PVN) of the hypothalamus controls the activity of the HPA axis. Totally, the limbic system regulates intrinsic and autonomic functions, as well as being involved in HPA axis regulation (1). Epilepsy impairs the function of the HPA axis (2). Its effect on the HPA axis can vary between generalized and localized epilepsies so that limbic seizures have stronger and greater effects than generalized seizures. According to previous studies, disruption in HPA axis affects the secretion level of the pituitary hormones resulting in changes in the concentration of these hormones in the blood (1). As epilepsy influences the hormones, the hormones can affect epilepsy. There is an intricate association in the corresponding relationship between epilepsy and such hormones. The effect of hormones on brain excitability can be illustrated by the frequencies of seizures $(3,4)$. Different sex hormones can be metabolized to different neurosteroids in the brain, so their effect is stronger on excitability. Considering the association between the temporolimbic system and the hypothalamus, epilepsy may modify sex hormones in both genders (5). A research surveying the differences in hormone levels between patients with left and right temporal lobe epilepsies indicated more secretion of gonadotropin-releasing hormone $(\mathrm{GnRH})$ in patients with left temporal lobe epilepsy (TLE) leading to an increased LH/FSH ratio and higher levels of testosterone (6). Another study investigated the level of $\mathrm{LH}$ in men with temporal epilepsy suggesting that the secretion of this hormone was confused (7). PRL level was measured after a seizure. It increased in all patients with generalized tonicclonic seizures, most patients with complex partial seizures and some with simple partial seizures (8). Therefore, the goal was to evaluate and compare the anterior pituitary hormones in these 2 types of epilepsy due to the difference in their origins and the corresponding relationship between hormones and epilepsy.

\section{Materials and Methods}

This cross-sectional study was conducted at Al-Zahra hospital of Isfahan, Iran, from spring 2014 to winter 2016. The population included all patients with the idiopathic first seizure, 36 persons of whom participated in the study by the consecutive method. The diagnosis of all patients was performed by a neurologist based on the International Classification of Seizures (9) and findings were consolidated based on electroencephalogram (EEG). Patients enrolled in this study included people aged over 18 with the first seizure of tonic-clonic epilepsy (TCE), or the first seizure of TLE referring within the first 24 hours of the seizure. In addition, the patients should not be pregnant, lactating female, postmenopausal, have Cushing's syndrome, be with hypothyroidism and hyperthyroidism, acromegaly, tuberous sclerosis (10), alcohol addicted, have brain tumor, be with chronic anxiety (11), with secondary generalized epilepsy, use hormonal contraceptive methods, be under the hormone replacement therapy, have a history of polycystic ovaries, have irregular menstrual syndrome and suffer from mesial temporal sclerosis. In addition, individuals treated with antiepileptic drugs, people with more than one seizure and those with reluctance to cooperate were excluded from the study. The data collection tool included a personal interview with the patient, an EEG and blood test. Demographic information of patients such as age, sex, marital status, level of education, and time of the seizure occurrence were collected via questionnaires. None of the patients were taking medicine.

Blood samples were obtained from all patients for measurement of anterior pituitary hormones involving FSH $(\mathrm{mIu} / \mathrm{mL}), \mathrm{LH}(\mathrm{mIu} / \mathrm{mL})$, ACTH $(\mathrm{pg} / \mathrm{mL}), \mathrm{GH}$ $(\mathrm{ng} / \mathrm{mL})$, TSH $(\mathrm{miclu} / \mathrm{mL})$ and PRL $(\mathrm{ng} / \mathrm{mL})$ with Diasorin kit and electrochemiluminescence device using ELISA method. Data analysis was performed through the SPSS software version 18.0 and descriptive and analytical methods using the $t$ test, chi-square test and Pearson correlation coefficient. The significance level for all statistical tests was considered 0.05 .

\section{Results}

The present study examined 36 patients with generalized and temporal lobe seizures, of whom 20 individuals had a generalized seizure and 16 suffered from temporal lobe seizure. The demographic characteristics of the patients are presented in Table 1.

About $53 \%$ of the patients in the tonic-clonic seizure group and $42 \%$ of the patients in the temporal lobe seizure group had normal EEGs.

The mean levels of FSH, ACTH, GH, PRL, LH, and TSH are reported in Table 2.

Overall, there was a significant relationship between seizure and GH $(P=0.005)$ and FSH $(P=0.033)$ levels, and no significant relation was found for LH, PRL, ACTH and TSH hormones.

The level of all anterior pituitary hormones other than TSH and ACTH in TLE was higher compared to TCE, but a significant difference was only observed in the levels of GH $(P=0.005)$ and FSH $(P=0.033)$. The levels of both hormones were significantly higher in the temporal lobe type than in the tonic-clonic type of epilepsy.

In TLE, PRL levels had a significant positive correlation with TSH $(P=0.007$ and $\mathrm{r}=0.649)$ and $\mathrm{ACTH}(P=$ 0.001 and $\mathrm{r}=0.753)$. In other words, an increment in PRL increases TSH and ACTH (Table 2).

In TCE, PRL levels had a significant positive correlation with TSH $(P=0.005$ and $\mathrm{r}=0.605)$ and FSH $(P=0.049$ and $\mathrm{r}=445)$ and $\operatorname{LH}(P=0.502$ and $\mathrm{r}=0.441)$. As a 
Table 1. Demographic characteristics of patients

\begin{tabular}{|c|c|c|c|c|c|}
\hline Characteristics & & Temporal lobe seizure & Tonic-clonic seizure & Seizure & $P$ value \\
\hline Age $($ mean $\pm S D)$ & & $32.43 \pm 9.2$ & $27.7 \pm 13.19$ & & 0.232 \\
\hline Sex & Male/female (\%) & $8(50 \%) / 8(50 \%)$ & $17(85 \%) / 3(15 \%)$ & $25(69.4 \%) / 11(30.6 \%)$ & 0.023 \\
\hline Marriage & Married/single (\%) & $9(56.3 \%) / 7(43.8 \%)$ & $9(45 \%) / 11(55 \%)$ & $18(50 \%) / 18(50 \%)$ & 0.502 \\
\hline Time of attack & Day/night (\%) & $12(75 \%) / 4(25 \%)$ & $9(47.4 \%) / 11(73.3 \%)$ & $21(58.4 \%) / 15(41.6 \%)$ & 0.096 \\
\hline \multirow{3}{*}{ Education } & Under diploma (\%) & $8(50 \%)$ & $9(45 \%)$ & $17(47.21 \%)$ & \multirow{3}{*}{0.216} \\
\hline & Diploma & $6(37.5 \%)$ & $5(25 \%)$ & $11(30.5 \%)$ & \\
\hline & College & $2(12.5 \%)$ & $6(30 \%)$ & $8(22.3 \%)$ & \\
\hline
\end{tabular}

Table 2. Comparison of the level of hormones in 2 types of tonic-clonic and temporal lobe epilepsies

\begin{tabular}{|c|c|c|c|c|c|c|}
\hline \multirow{3}{*}{ Hormones } & \multicolumn{4}{|c|}{ Seizure } & \multirow{3}{*}{ Normal range } & \multirow{3}{*}{$P$ value } \\
\hline & \multicolumn{2}{|c|}{ Tonic-clonic } & \multicolumn{2}{|c|}{ Temporal lobe } & & \\
\hline & Number & Mean $\pm S D$ & Number & Mean \pm SD & & \\
\hline TSH & 20 & $2.96 \pm 3.31$ & 16 & $2.15 \pm 1.1$ & $0.4-4.6$ & 0.355 \\
\hline $\mathrm{GH}$ & 20 & $1.87 \pm 2.55$ & 16 & $4.59 \pm 2.95$ & Up to 14 & 0.005 \\
\hline АСТН & 20 & $33.47 \pm 17.67$ & 16 & $23.7 \pm 20.7$ & Up to 71 & 0.136 \\
\hline PRL & 20 & $14.15 \pm 8.81$ & 16 & $15.42 \pm 16.61$ & $2-24$ & 0.77 \\
\hline FSH & 20 & $4.34 \pm 2.37$ & 16 & $14.06 \pm 19.4$ & $1-7$ & 0.033 \\
\hline $\mathrm{LH}$ & 20 & $6.19 \pm 5.56$ & 16 & $9.37 \pm 6.18$ & $1-8$ & 0.116 \\
\hline
\end{tabular}

Abbreviations: TSH, thyroid-stimulating hormone; $\mathrm{ACTH}$, adrenocorticotrophic hormone; $\mathrm{GH}$, growth hormone; FSH, follicle-stimulating hormone, $\mathrm{LH}$, luteinizing hormone, PRL, prolactin.

matter of fact, PRL increment leads to an increase in TSH, FSH and LH levels.

\section{Discussion}

In this study, there was a significant relationship between seizure and levels of GH and FSH hormones, and no significant relationship was found between the other hormones.

The level of all hormones other than TSH and ACTH was high in the temporal lobe seizure than in the tonicclonic seizure, but a significant difference was observed only in the levels of the GH and FSH. The levels of both hormones were significantly higher in the temporal lobe type than in the TCE.

The anterior pituitary is often known as the master gland since it organizes and coordinates the regulation of other endocrine glands, along with the hypothalamus. Pituitary hormones are secreted by a pulsing pattern, indicating that they are stimulated by a series of hypothalamic releasing factors. Temporal lobe seizure originates from the temporal lobe area which plays an important role in organizing sensory inputs as well as in auditory, lingual, and verbal comprehension.

The hippocampus, parahippocampus, amygdala, and hypothalamus are parts of the limbic system located in the temporal lobe. Amygdala is an area that has a bilateral relationship with many brain regions, including thalamus, hypothalamus, hippocampus, parahippocampus, and brain stem. The amygdala is an essential area for coordinating behaviors and autonomic and intrinsic response to environmental stimuli (especially with emotional content) and stimulates the secretion of glucocorticoids. Therefore, damage to the amygdala reduces the response to emotional stimuli. The hippocampus receives sensory inputs from the neocortex, especially the limbic cortex, amygdala, and hypothalamus. Temporal lobe seizure typically originates from this area. Hippocampus inhibits the activity of the HPA axis. The hypothalamus receives information from the hippocampus and the amygdala, and the PVN of hypothalamus controls the activity of the HPA axis, which has a direct relationship with the limbic system and the PVN. Furthermore, the limbic system adjusts the autonomic and intrinsic function, especially in response to emotional stimuli. This system is also involved in regulating the HPA axis (1).

In patients with epilepsy, the base level of stress hormones increases, which can increase subsequent seizures. Epilepsy impairs the regulation of the HPA axis function (2). The effect of epilepsy on HPA can be different in generalized and localized epilepsies. Limbic system seizures have more powerful and bigger effects than generalized seizures (1). Activation of HPA by initial seizure may be related to subsequent seizures (2). Therefore, it is reasonable that the differences between levels of hormones were observed in both types of seizures in this study and other findings are in accordance with that.

A research studied FSH levels in 33 male patients with seizure and 11 healthy individuals in the control group. 
The level of FSH in patients significantly increased (12). In another study, the level of this hormone was measured in 2 types of epilepsy, which increased only in female participants (13).

The brain controls the reproductive function by regulating the hypothalamus in the pituitary gland (14). The areas of the hypothalamus, involved in GnRH secretion and regulation, receive extensive direct communication from the cerebral hemispheres, especially the temporolimbic structures and amygdale $(12,15)$. The temporal lobe seizure increases the frequency of $\mathrm{GnRH}$ secretions, which is associated with an increase in the LH/ FSH ratio. Different sex hormones can be metabolized into different neurosteroids in the brain and their effect on excitability is stronger. Estrogen further stimulates the development of epilepsy, while progesterone has an antiepileptic effect.

Fluctuations in hormonal levels, especially in estrogen and progesterone levels, are associated with changes in seizure patterns. These hormones seem not to be the primary cause of seizure but can alter the pattern of seizures. They can also affect brain function by disturbing specific hormonal receptors that affect the neurons. The high density of these receptors is seen in areas that are important in causing seizures (12). In a study, Premarin, an injectable estrogen, was injected into 16 women with epilepsy, by which seizure increased in 11 of them. Compared with estrogen, progesterone injections in people with epilepsy reduced their seizures (16).

Testosterone and its effect on seizure depend on its metabolites. Estradiol and 3- $\alpha$-androstenediol stimulate seizure. Dihydrotestosterone reduces neuronal excitability (12).

In a study on 49 selected patients suffering from one of these 2 types of epilepsy, regardless of gender, the level of GH increased in some patients with TCE but the level of this hormone did not significantly increase in subjects with complex partial epilepsy (17).

Another study measured the level of GH in 12 patients with both types of epilepsy and 28 healthy cases in the control group. This study showed that the amount of GH increased following seizures (18).

The serum level of ghrelin (a hormone secreted mainly from the stomach) increases in the case of epilepsy. When ghrelin enters the nervous system via a blood-brain barrier, it enhances the GH gene expression in the hippocampus and the cortex leads to increased secretion (19).

Comparison of PRL levels of serum in 2 groups of epilepsy in previous studies showed that serum PRL levels had more increment in TLE (13). In another study, serum PRL was examined in more than 500 seizures which increased in $88 \%$ of tonic-clonic seizures and $78 \%$ of temporal lobe seizures (20). The factor that contributed to differences in the results of PRL in this study and other studies was that the time constraints for blood sampling were not considered, while other studies have regarded specific time for sampling.

In a study on patients with temporal lobe and tonicclonic epilepsies, higher levels of ACTH secretion were observed after epileptic seizures (21).

An increase in LH levels was observed in a study on women with multiple epileptic seizures without consuming antiepileptic drugs (22).

A research that measured the level of TSH in patients with epilepsy and control group showed no difference between the affected and healthy subjects (23).

Another research compared TSH levels in 88 patients with seizure and in 30 healthy individuals in control group, where and the level of this hormone was reported to be high in $4 \%$ of the patients (24).

Patients in this study referred after the first seizure and their number was limited, while individuals with chronic epilepsy were selected in other studies. This may cause some differences compared to previous studies.

The strength of our study was examining patients with the first epileptic seizure. These patients did not take any medication.

Antiepileptic drugs also cause hormonal changes, because they, directly and indirectly, affect the function of the HPA axis (25). These drugs accelerate the metabolism of hormones by inducing effects on the liver enzymes and reducing their serum concentrations. Antiepileptic drugs can alter endocrine function in both genders with epilepsy, and this change can cause significant reproductive disturbances (12).

\section{Conclusion}

The current study found a significant relationship between seizure and levels of GH and FSH hormones, and no significant relationship was observed with other hormones.

The level of all hormones except TSH and ACTH was higher in temporal lobe seizure than in tonic-clonic seizure, but there was a significant difference only in the level of GH and FSH. The level of both hormones in the temporal lobe type is significantly higher compared to TCE. Extensive studies along with more patients are required to consolidate these findings.

\section{Conflict of interests \\ None.}

Ethical considerations

The study was approved by the ethics committee of Isfahan University Of Medical Sciences (No. 293057).

\section{Acknowledgments}

We appreciate the staff of Al-Zahra hospital and university professors who collaborated in this research (Research code: 293057).

\section{References}

1. Maguire J, Salpekar JA. Stress, seizures, and hypothalamicpituitary-adrenal axis targets for the treatment of epilepsy. Epilepsy Behav. 2013;26(3):352-62. doi: 10.1016/j. 
yebeh.2012.09.040.

2. O'Toole KK, Hooper A, Wakefield S, Maguire J. Seizureinduced disinhibition of the HPA axis increases seizure susceptibility. Epilepsy Res. 2014;108(1):29-43. doi: 10.1016/j.eplepsyres.2013.10.013.

3. Herzog AG. Catamenial epilepsy: Update on prevalence, pathophysiology and treatment from the findings of the NIH Progesterone Treatment Trial. Seizure. 2015;28:18-25. doi: 10.1016/j.seizure.2015.02.024.

4. Tauboll E, Roste LS, Svalheim S. Catamenial Epilepsy. In: Harden CL, Thomas SV, Tomson T, eds. Epilepsy in Women. UK: John Wiley \& Sons Ltd; 2013:41-52.

5. Tauboll E, Sveberg L, Svalheim S. Interactions between hormones and epilepsy. Seizure. 2015;28:3-11. doi: 10.1016/j. seizure.2015.02.012.

6. Herzog AG, Coleman AE, Jacobs AR, Klein P, Friedman MN, Drislane FW, et al. Relationship of sexual dysfunction to epilepsy laterality and reproductive hormone levels in women. Epilepsy Behav. 2003;4(4):407-13.

7. Quigg M, Kiely JM, Johnson ML, Straume M, Bertram EH, Evans WS. Interictal and postictal circadian and ultradian luteinizing hormone secretion in men with temporal lobe epilepsy. Epilepsia. 2006;47(9):1452-9. doi: 10.1111/j.15281167.2006.00617.x.

8. Morrell MJ, Montouris GD. Reproductive disturbances in patients with epilepsy. Cleve Clin J Med. 2004;71 Suppl 2:S19-24.

9. Fisher RS, Cross JH, French JA, Higurashi N, Hirsch E, Jansen $\mathrm{FE}$, et al. Operational classification of seizure types by the International League Against Epilepsy: Position Paper of the ILAE Commission for Classification and Terminology. Epilepsia. 2017;58(4):522-30. doi: 10.1111/epi.13670.

10. Saxena A, Sampson JR. Epilepsy in tuberous sclerosis: phenotypes, mechanisms, and treatments. Semin Neurol. 2015;35(3):269-76. doi: 10.1055/s-0035-1552616.

11. Dube CM, Molet J, Singh-Taylor A, Ivy A, Maras PM, Baram TZ. Hyper-excitability and epilepsy generated by chronic early-life stress. Neurobiol Stress. 2015;2:10-9. doi: 10.1016/j. ynstr.2015.03.001.

12. Rodin E, Subramanian MG, Gilroy J. Investigation of sex hormones in male epileptic patients. Epilepsia. 1984;25(6):690-4.

13. Dana-Haeri J, Trimble M, Oxley J. Prolactin and gonadotrophin changes following generalised and partial seizures. J Neurol Neurosurg Psychiatry. 1983;46(4):331-5.
14. Thompson IR, Kaiser UB. GnRH pulse frequency-dependent differential regulation of $\mathrm{LH}$ and $\mathrm{FSH}$ gene expression. Mol Cell Endocrinol. 2014;385(1-2):28-35. doi: 10.1016/j. mce.2013.09.012.

15. Marques P, Skorupskaite K, Rozario KS, Anderson RA, George JT. Physiology of $\mathrm{GnRH}$ and gonadotropin secretion. In: In: Feingold KR, Anawalt B, Boyce A, Chrousos G, Dungan K, Grossman A, eds. Endotext . South Dartmouth (MA): MDText. com, Inc.; 2015.

16. Backstrom T, Zetterlund B, Blom S, Romano M. Effects of intravenous progesterone infusions on the epileptic discharge frequency in women with partial epilepsy. Acta Neurol Scand. 1984;69(4):240-8.

17. Takeshita H, Kawahara R, Nagabuchi T, Mizukawa R, Hazama H. Serum prolactin, cortisol and growth hormone concentrations after various epileptic seizures. Jpn J Psychiatry Neurol. 1986;40(4):617-23.

18. Rao ML, Stefan H, Bauer J. Epileptic but not psychogenic seizures are accompanied by simultaneous elevation of serum pituitary hormones and cortisol levels. Neuroendocrinology. 1989;49(1):33-9. doi: 10.1159/000125088.

19. Khatib N, Gaidhane S, Gaidhane AM, Khatib M, Simkhada P, Gode D, et al. Ghrelin: ghrelin as a regulatory Peptide in growth hormone secretion. J Clin Diagn Res. 2014;8(8):Mc137. doi: $10.7860 / j c d r / 2014 / 9863.4767$.

20. Bilo L, Meo R, Nappi C, Annunziato L, Striano S, Colao AM, et al. Reproductive endocrine disorders in women with primary generalized epilepsy. Epilepsia. 1988;29(5):612-9.

21. Gallagher BB. Endocrine abnormalities in human temporal lobe epilepsy. Yale J Biol Med. 1987;60(2):93-7.

22. Bilo L, Meo R, Valentino R, Buscaino GA, Striano S, Nappi C. Abnormal pattern of luteinizing hormone pulsatility in women with epilepsy. Fertil Steril. 1991;55(4):705-11.

23. Zhu SQ, Liu XM, Ruan XZ, Cai Z. Changes of thyroid hormone levels in epileptic patients. J Tongji Med Univ. 1994;14(2):11923.

24. Hamed SA, Hamed EA, Kandil MR, El-Shereef HK, Abdellah MM, Omar H. Serum thyroid hormone balance and lipid profile in patients with epilepsy. Epilepsy Res. 2005;66(13):173-83. doi: 10.1016/j.eplepsyres.2005.08.004.

25. Verrotti A, D’Egidio C, Mohn A, Coppola G, Parisi P, Chiarelli F. Antiepileptic drugs, sex hormones, and PCOS. Epilepsia. 2011;52(2):199-211. doi: 10.1111/j.15281167.2010.02897.x. 